\title{
Response rate and diagnostic accuracy of early PET-CT during neo-adjuvant therapies in oesophageal adenocarcinoma: a systematic review and meta-analysis
}

\author{
Kieran Foley ${ }^{1}$, Jacqueline Jeffries ${ }^{2}$, Clare Hannon ${ }^{3}$, Bernadette Coles ${ }^{1}$, Kevin Bradley ${ }^{4}$, and \\ Elizabeth Smyth ${ }^{3}$ \\ ${ }^{1}$ Velindre Cancer Centre \\ ${ }^{2}$ National Imaging Academy Wales (NIAW) \\ ${ }^{3}$ Cambridge University Hospitals NHS Foundation Trust \\ ${ }^{4}$ Wales Diagnostic and Research Positron Emission Tomography Imaging Centre (PETIC)
}

October 22, 2020

\begin{abstract}
Purpose Only $25 \%$ of oesophageal adenocarcinoma (OAC) patients have a pathological response to neo-adjuvant therapy (NAT) before oesophagectomy. Early response assessment using PET imaging may help guide management of these patients. We performed a systematic review and meta-analysis to synthesise the evidence detailing response rate and diagnostic accuracy of early PET-CT assessment. Methods We systematically searched several databases including MEDLINE and Embase. Studies with mixed cohorts of histology, tumour location, and a repeat PET-CT assessment after more than one cycle of NAT were excluded. Reference standard was pathological response, defined by Becker or Mandard classifications. Primary outcome was metabolic response rate after one cycle of NAT defined by a reduction in maximum standardised uptake value (SUVmax) of $35 \%$. Secondary outcome was diagnostic accuracy of treatment response prediction, defined as the sensitivity and specificity of early PET-CT using this threshold. Quality of evidence was also assessed. Random-effects meta-analysis pooled response rates and diagnostic accuracy. This study was registered with PROSPERO (CRD42019147034). Results Overall, 1341 articles were screened, and six studies were eligible for analysis. These studies reported data for 518 patients (aged 27-78 years; 452 [87.3\%] were male) between 2005-2020. Pooled sensitivity of early metabolic response to predict pathological response was $77.2 \%$ (95\%CI 53.2\%-100\%). Significant heterogeneity existed between studies (I2=80.6\% (95\%CI 38.9\%-93.8\%), p=0.006). Pooled specificity was $75.0 \%$ (95\% CI $68.2 \%-82.5 \%$ ), however no significant heterogeneity between studies existed (I2=0.0\% (95\% CI $0.0 \%-67.4 \%), \mathrm{p}=0.73)$. Conclusion High-quality evidence is lacking, and few studies met the inclusion criteria of this systematic review. The sensitivity of PET using a SUVmax reduction threshold of $35 \%$ was suboptimal and varied widely. However, specificity was consistent across studies with a pooled value of $75.0 \%$, suggesting early PET assessment is a better predictor of treatment resistance than of pathological response. Further research is required to define optimal PET-guided treatment decisions in OAC.
\end{abstract}

\section{Title Page}

Response rate and diagnostic accuracy of early PET-CT during neo-adjuvant therapies in oesophageal adenocarcinoma: a systematic review and meta-analysis

Short Title: Early PET-CT in oesophageal adenocarcinoma

Kieran G Foley ${ }^{1 *}$, Jacqueline Jeffries ${ }^{2}$, Clare Hannon ${ }^{3}$, Bernadette Coles ${ }^{1}$, Kevin M Bradley ${ }^{4}$, Elizabeth Smyth $^{3}$ 
1 Velindre Cancer Centre, Cardiff, UK

2 National Imaging Academy of Wales (NIAW), Pencoed, UK

3 Cambridge University Hospitals NHS Foundation Trust, Cambridge, UK

4 Wales Diagnostic and Research Positron Emission Tomography Imaging Centre, Cardiff University, UK

* corresponding author

Dr Kieran Foley, Velindre Cancer Centre, Velindre Road, Cardiff, CF14 2TL

Email: Kieran.Foley@wales.nhs.uk

\section{Acknowledgements}

No funding was received for this work.

The authors have no conflicts of interest to declare.

\section{Hosted file}

Main Text.pdf available at https://authorea.com/users/369341/articles/488232-response-rateand-diagnostic-accuracy-of-early-pet-ct-during-neo-adjuvant-therapies-in-oesophagealadenocarcinoma-a-systematic-review-and-meta-analysis

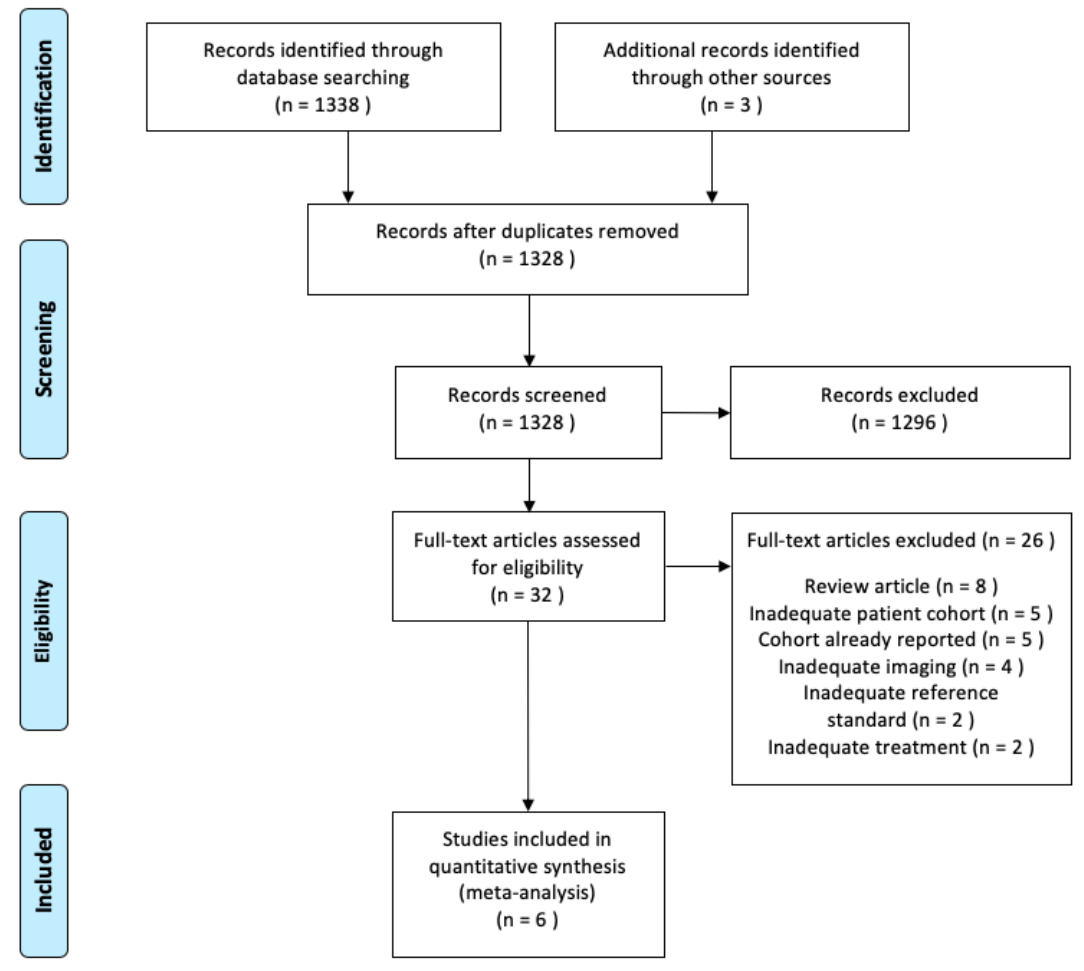




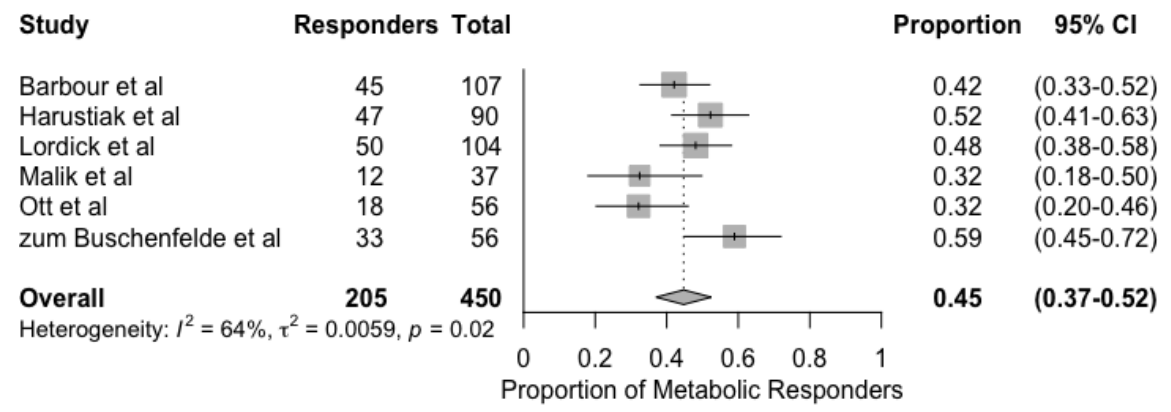

A

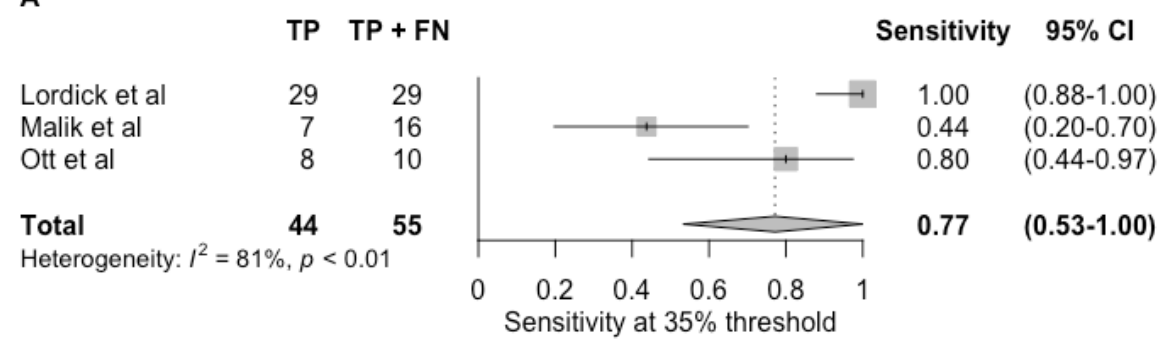

B

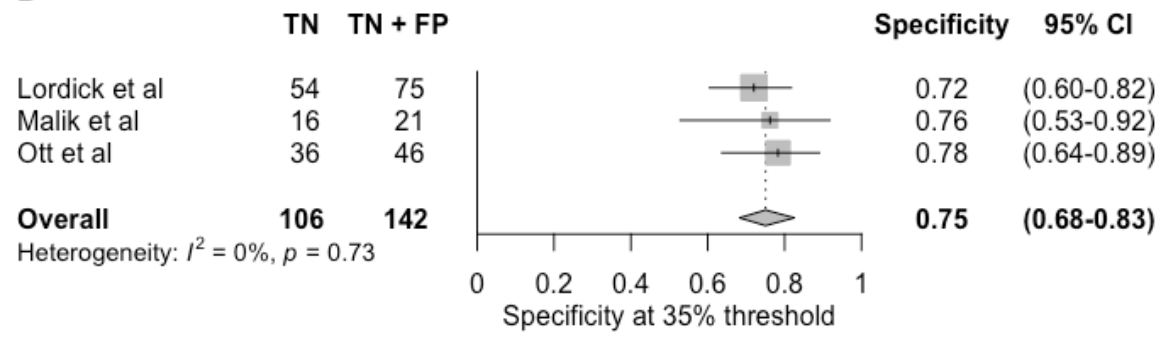

